\title{
BMC Genetics reviewer acknowledgement 2015
}

Julia Simundza

\section{Contributing reviewers}

The editors of BMC Genetics would like to thank all our reviewers who have contributed to the journal in Volume 16 (2015).

Adam Lukaszewski
USA

Vishal Acharya

India

Vera Afreixo

Portugal

Jon Ahlinder

Sweden

Kwangmi Ahn

USA

Omar Albagha

UK

Florian Alberto

France

John Alcorn

USA

Emil Alexov

USA

Kristina Allen-Brady

USA

Santos Alonso

Spain

Stefan Ambs

USA

Satyanarayana Ande

USA
Magnus Andersson
Finland

Niki Antypa

Netherlands

Juan Jose Arranz

Spain

Jennifer Asimit

UK

John Attia

Australia

Antonios Augustinos

Greece

Frédéric Austerlitz

France

Jeremy Austin

Australia

David Aylor

USA

Hela Azaiez

USA

Yvonne Badke

Belgium

Ernest Bailey

USA

Diane Bailleul

France

\author{
Lorenzo Barchi \\ Italy \\ Esther Becker \\ UK
}

Albano Beja-Pereira
Portugal
Daniele Bellavia
Italy
Olga Beltcheva
Bulgaria

Rosaria Benedetti

Italy

Tillmann Benfey

Canada

\section{Brian Bennett}

USA

Kerin Bentley

USA

\section{Peer Berg}

Norway

\author{
Justo Lorenzo Bermejo \\ Germany \\ Donagh Berry \\ Ireland
}

Nils-Ove Bertholdsson

Sweden 
Viviana Berthoud

USA

Etienne Bezault

USA

Maria Assunta Biscotti

Italy

Didier Boichard

USA

Folmer Bokma

Sweden

Carolina Bonilla

UK

Marina Bonin

Brazil

Lara Bossini-Castillo

UK

Aniek C. Bouwman

Netherlands

Carine Brouat

France

Louise Brousseau

France

Jácome Bruges-Armas

Portugal

Su-Hong Bu

China

Bart Buitenhuis

Denmark

Cynthia Bulik

USA

Jarek Burczyk

Poland

Lei Cai

China

Yafei Cai

China

Mario Calus

Netherlands

Xiaoyi Cao

USA

Eduardo Casas

USA

Sergi Castellano

Germany
Hugo Ten Cate

Netherlands

Carlos Celis-Morales

UK

Lisa Chakrabarti

UK

Ken Chalmers

USA

Hua Chen

China

Hong Chen

China

Charles Y. Chen

USA

Jianli Chen

USA

Piedu Chen

China

Wei-Min Chen

USA

Yizhou Chen

Australia

Vikram Chhatre

USA

Yoon Shin Cho

South Korea

Bruno Conti

USA

Ernest Gus Cothran

USA

Eliecer Coto

Spain

Vincent Coustham

France

Nigel Crawford

USA

Marion Cremer

Germany

Cheryl Cropp

USA

Yuehua Cui

USA

Song Cui

USA
Tatiana Danilova

USA

Andrew Davie

UK

M. Davis

USA

Tamara Davis

USA

Michelle Daya

South Africa

Mariza De Andrade

USA

Maria Luisa Dettori

Italy

H.S. Dhaliwal

India

Xianmin Diao

China

Alessandro Didonna

USA

Mark Dopson

Sweden

Mehmet Tevfik Dorak

UK

Sami Dridi

USA

Britt Drögemöller

Canada

Qingzhang Du

China

Naomi Duijvesteijn

Netherlands

Yann Dussert

France

Craig Echt

USA

Vahid Edriss

Denmark

John Edwards

USA

Darrell Ellsworth

USA

Rachel Ellsworth

USA 
Ingegerd Elvers

USA

Malena Erbe

Germany

Bruno Fady

France

Robert Feil

France

Bingjian Feng

USA

Jane Ferguson

USA

Jose Fernandez

USA

Susana Ferreira

Portugal

Martin Ferris

USA

Martin Flajshans

Czech Republic

Burkhardt Flemer

Ireland

Richard Fletcher

USA

Carlos Flores

Spain

Jia Nee Foo

Singapore

Marina Fortes

Australia

Giuliana Fortunato

Italy

Breno Fragomeni

USA

Wenqing Fu

USA

Maria Fuciarelli

Italy

Silvia Fuselli

Italy

Agata Gadaleta

Italy

Andrea Gaedigk

USA
Mercedes Garayoa

Spain

Christiane Gebhardt

Germany

Phil Giffard

Australia

Elizabeth Gilbert

USA

Tania Gioia

Germany

Ian Gizer

USA

Andreas Gogol-Doering

Germany

Pengtao Gong

China

George Goulielmos

Greece

Manje Gowda

India

Surendra Goyal

India

Paul Grabowski

USA

Cecile Grenier

Colombia

Ben Greyling

South Africa

Thomas Gridley

USA

Darren Griffin

UK

Daniel Grinberg

USA

Vince Kornél Grolmusz

Hungary

Christine Große-Brinkhaus

Germany

David Groth

Australia

Xing-You Gu

USA

Rudy Guerra

USA
Carlos Guerrero-Bosagna

Sweden

Paul Gugger

USA

Longbiao Guo

China

Yunqian Guo

China

Shicheng Guo

USA

Sanjeev Gupta

Ireland

Jenny Hagenblad

Sweden

M Haile-Mariam

Australia

David Haldane

Canada

Jianlin Han

Kenya

Lee Harold

USA

Liang $\mathrm{He}$

Finland

Meian He

China

Xinhua He

China

Xin He

USA

Tao He

USA

Scott Hebbring

USA

Chew Kiat Heng

Singapore

Julie Hicks

USA

André Hidalgo

Netherlands

Emmeline Hill

Ireland

Katrin Hinderhofer

Germany 
Lies Hoefsloot

Netherlands

\section{Gabriel Hoffman}

USA

Josephine Hoh

USA

Daniela Holtgräwe

Germany

Ian Hope

UK

Jian Hou

China

Liping Hou

USA

Guo $\mathrm{Hu}$

China

Jie Hu

USA

Yijuan $\mathrm{Hu}$

USA

Xu Hua

China

Lei Huang

China

Wei Huang

China

Kai Huang

USA

Hailiang Huang

USA

Wen Huang

USA

David Hughes

Spain

Stefanie Huhn

Germany

György Hutvágner

Australia

Kamal Ibrahim

USA

Meryem Ipek

Turkey

Daniel Irimia

USA
Brian Irish

USA

Akira Ishikawa

Japan

Jeanne Jacobs

New Zealand

Malgorzata Jankun

Poland

Luc Janss

Denmark

Yulin Jia

USA

Xueqiu Jian

USA

Bruce Johnson

USA

Jenny Juengel

New Zealand

Haja Kadarmideen

Denmark

Lukáš Kalous

Czech Republic

Guolian Kang

USA

Juha Kantanen

Finland

Rebecca Kartzinel

USA

Jaebum Kim

Republic Of Korea

Jung Han Kim

USA

Elizabeth King

USA

Rick Kittles

USA

Christoph Knorr

Germany

Michael Hans Kohn

USA

Caline Koh-Tan

UK

Roland Kölliker

Switzerland
Willem Kruijer

Netherlands

Haydn Kuchel

Australia

Santosh Kumar

Canada

Jaya Kumari

Norway

Vanisha Lakhina

USA

Denis Laloe

France

Hovirag Lancioni

Italy

Nicholas Larson

USA

Sabrina Le Cam

France

Ju-Seog Lee

USA

Seunggeun Lee

USA

Sigrid Lehnert

Australia

Gabriel Leitner

Israel

Johannes Lenstra

Netherlands

Chun Li

China

Ao Li

China

Hui Li

China

Zhang Li

China

Qigang Li

China

Zitong Li

Finland

Jun Li

USA

Zichao Li

China 
Yuqing Li

USA

Yi Li

Singapore

Xiaoxi Lin

Canada

Xu Lin

China

Gabriella Lindgren

Sweden

Amanda Lindholm-Perry

Mugen Liu

China

Xu Liu

China

Bin Liu

China

Jianquan Liu

China

Wansheng Liu

USA

Zengting Liu

Germany

Adrian Llerena

Spain

Stephanie London

USA

Saioa Lopez

Spain

Siew Low

Australia

Lizhi Lu

China

Xinhong Luan

China

Pierre Luisi

Spain

Adam Lukaszewski

USA

Keming Luo

China

Ma Luo

Canada
Jiangtao Luo

USA

Guansheng Ma

China

Xiaochun Ma

China

Peipei Ma

China

Steffen Maak

Germany

Margaret Mackinnon

Kenya

Panagiotis Madesis

Greece

Laurence Maréchal-Drouard

France

Vladmir Margarido

Brazil

Osvaldo Marinotti

USA

Donata Marletta

Italy

Kathleen Martin

USA

Cesar Martins

Brazil

Scot Matkovich

USA

Taylor Maxwell

USA

James Mccreight

USA

Molly Mccue

USA

Michael Mcgeachie USA

J. Mitchell Mcgrath USA

Kieran Meade

Ireland

Jennifer Meadows

Sweden

Hendrik-Jan Megens

Netherlands
Andreas Menke

Germany

Susan Meyer

USA

Phil Miklas

USA

Dan Milbourne

Ireland

Cecelia Miles

USA

Gabriel Minarik

USA

Juan Miranda-Rios

Mexico

Suresh Mishra

Canada

Xavier Montagutelli

France

Christopher Moran

Australia

Gota Morota

USA

Jeong-Hwan Mun

South Korea

Steven Munger

USA

Moses Muraya

Germany

Hideki Mutai

Japan

Teruyoshi Nagamitsu

Japan

Hugo Naya

Uruguay

Christopher Nelson

UK

Frank Nicholas

Australia

Garth Nicholson

Australia

Motohide Nishio

Japan

Dan Nonneman

USA 
Johannes Novak

Austria

Domenico Nuzzo

Italy

Thomas O'Brien

USA

Jorgen Odegard

Norway

Thomas Odong

Netherlands

Junko Okano
Japan
Patricia Okubara
USA

Jose L. Oliver

Spain

Angélica Olivo-Díaz

Mexico

Ettore Olmo

Italy

Hanne Gro Olsen

Norway

Bode Olukolu

USA

Akio Onogi

Japan

Tamás Orbán

Hungary

Pablo Orozco-Terwengel

UK

Richard Orrell

UK

Wei Pan

USA

Manish Pandey

India

Bibhusita Pani

USA

Afshin Parsa

USA

Hemal Patel

USA

Hubert Pausch

Germany
Michele Pazzola

Italy

Jason Peart

Australia

Jill Pecon-Slattery

USA

Niels Pedersen

USA

Francisco Peñagaricano USA

Hans-Peter Piepho

Germany

Marija Pljesa-Ercegovac

Serbia

Andrea Porceddu

Italy

Manoj Prasad

India

Marcin Pszczola

Poland

Deirdre C Purfield

Ireland

Xinshuai Qi

USA

Wanqiong Qiao

USA

Padmalatha Rai

India

G Ravikanth

India

Sarah Rea

Australia

James Reecy

USA

Monika Reißmann

Germany

Romdhane Rekaya

USA

Jun Ren

China

Pascal Rihet

France

David Riley

USA
Isabel Rivera

Portugal

James Roberds

USA

Christèle Robert-Granié

France

Dale Roberts

Australia

Monica Rodriguez

Italy

Juan Rodriguez-Flores

USA

Claire Rogel-Gaillard

France

Megan Rolf

USA

Zsolt Ronai

Hungary

Stephanie Rosse

USA

Florence Rouleux-Bonnin

France

Yuefeng Ruan

Canada

Sachin Rustgi

USA

Peter Ryan

Australia

Mahdi Saatchi

USA

Francois Sabot

France

Giuseppe Saccone

Italy

Wolfgang Sadee

USA

Goutam Sahana

Denmark

Srinivas Vinod Saladi

USA

Lucas Sanchez

Spain

Juliano Sangalli

Brazil 


\begin{tabular}{|c|c|c|}
\hline $\begin{array}{l}\text { Miguel Santana } \\
\text { USA }\end{array}$ & $\begin{array}{l}\text { Mukerjee Shomita } \\
\text { India }\end{array}$ & $\begin{array}{l}\text { Charles Steward } \\
\text { UK }\end{array}$ \\
\hline $\begin{array}{l}\text { Pratik Satya } \\
\text { India }\end{array}$ & $\begin{array}{l}\text { Daniel Shriner } \\
\text { USA }\end{array}$ & $\begin{array}{l}\text { Paul Stothard } \\
\text { Canada }\end{array}$ \\
\hline $\begin{array}{l}\text { Ana Savic-Radojevic } \\
\text { Serbia }\end{array}$ & $\begin{array}{l}\text { Chang Shu } \\
\text { USA }\end{array}$ & $\begin{array}{l}\text { Patrick Stratz } \\
\text { Germany }\end{array}$ \\
\hline $\begin{array}{l}\text { Ruairidh Sawers } \\
\text { Mexico }\end{array}$ & $\begin{array}{l}\text { Animesh Shukla } \\
\text { USA }\end{array}$ & $\begin{array}{l}\text { Guosheng Su } \\
\text { Denmark }\end{array}$ \\
\hline $\begin{array}{l}\text { Ian Sayers } \\
\text { UK }\end{array}$ & $\begin{array}{l}\text { Sanjeev Shukla } \\
\text { India }\end{array}$ & $\begin{array}{l}\text { Kazuhiko Sugimoto } \\
\text { Japan }\end{array}$ \\
\hline $\begin{array}{l}\text { Joseph Schacherer } \\
\text { France }\end{array}$ & $\begin{array}{l}\text { Timothy Shutt } \\
\text { Canada }\end{array}$ & $\begin{array}{l}\text { G Suman Latha } \\
\text { India }\end{array}$ \\
\hline $\begin{array}{l}\text { Flávio Schenkel } \\
\text { Canada }\end{array}$ & $\begin{array}{l}\text { Katja Silbermayr } \\
\text { Austria }\end{array}$ & $\begin{array}{l}\text { Fanyue Sun } \\
\text { USA }\end{array}$ \\
\hline $\begin{array}{l}\text { Antje Schierholt } \\
\text { Germany }\end{array}$ & $\begin{array}{l}\text { Xueling Sim } \\
\text { Singapore }\end{array}$ & $\begin{array}{l}\text { Tatiana Suslova } \\
\text { Russian Federation }\end{array}$ \\
\hline $\begin{array}{l}\text { Christian Schlötterer } \\
\text { Austria }\end{array}$ & $\begin{array}{l}\text { Magda Sindicic } \\
\text { Croatia }\end{array}$ & $\begin{array}{l}\text { Nicolas Sylvius } \\
\text { UK }\end{array}$ \\
\hline $\begin{array}{l}\text { Jennifer Schmidt } \\
\text { USA }\end{array}$ & $\begin{array}{l}\text { Shree Ram Singh } \\
\text { USA }\end{array}$ & $\begin{array}{l}\text { Joanna Szyda } \\
\text { Poland }\end{array}$ \\
\hline $\begin{array}{l}\text { Armin Schmitt } \\
\text { Italy }\end{array}$ & $\begin{array}{l}\text { Johanna Sistonen } \\
\text { Switzerland }\end{array}$ & $\begin{array}{l}\text { Hidenori Tachida } \\
\text { Japan }\end{array}$ \\
\hline $\begin{array}{l}\text { Andreas Schmitz } \\
\text { Switzerland }\end{array}$ & $\begin{array}{l}\text { Loren Skow } \\
\text { USA }\end{array}$ & $\begin{array}{l}\text { Kobi Tadmor } \\
\text { Israel }\end{array}$ \\
\hline $\begin{array}{l}\text { Robert Schmitz } \\
\text { USA }\end{array}$ & $\begin{array}{l}\text { Tony Slater } \\
\text { Australia }\end{array}$ & $\begin{array}{l}\text { Fasil Tekola-Ayele } \\
\text { USA }\end{array}$ \\
\hline $\begin{array}{l}\text { Dustin Schones } \\
\text { USA }\end{array}$ & $\begin{array}{l}\text { Petr Smykal } \\
\text { Czech Republic }\end{array}$ & $\begin{array}{l}\text { Raimund Tenhaken } \\
\text { Austria }\end{array}$ \\
\hline $\begin{array}{l}\text { Britta Schulz } \\
\text { Germany }\end{array}$ & $\begin{array}{l}\text { Eric Snyder } \\
\text { USA }\end{array}$ & $\begin{array}{l}\text { Jens Tetens } \\
\text { Germany }\end{array}$ \\
\hline $\begin{array}{l}\text { Hervé Seligmann } \\
\text { France }\end{array}$ & $\begin{array}{l}\text { Leah Solberg Woods } \\
\text { USA }\end{array}$ & $\begin{array}{l}\text { Sophie Thevenon } \\
\text { France }\end{array}$ \\
\hline $\begin{array}{l}\text { Nick Serão } \\
\text { USA }\end{array}$ & $\begin{array}{l}\text { Qingxuan Song } \\
\text { USA }\end{array}$ & $\begin{array}{l}\text { Vicki Thomson } \\
\text { Australia }\end{array}$ \\
\hline $\begin{array}{l}\text { Andrey Shcherban } \\
\text { Russian Federation }\end{array}$ & $\begin{array}{l}\text { Chi Song } \\
\text { USA }\end{array}$ & $\begin{array}{l}\text { Francesco Tiezzi } \\
\text { USA }\end{array}$ \\
\hline $\begin{array}{l}\text { Amir Sherman } \\
\text { Israel }\end{array}$ & $\begin{array}{l}\text { Pavel Souček } \\
\text { Czech Republic }\end{array}$ & $\begin{array}{l}\text { Peter Tiffin } \\
\text { USA }\end{array}$ \\
\hline $\begin{array}{l}\text { Clive Shiff } \\
\text { USA }\end{array}$ & $\begin{array}{l}\text { Leon Spicer } \\
\text { USA }\end{array}$ & $\begin{array}{l}\text { Alex Todorov } \\
\text { USA }\end{array}$ \\
\hline $\begin{array}{l}\text { Masayoshi Shigyo } \\
\text { Japan }\end{array}$ & $\begin{array}{l}\text { James Squires } \\
\text { Canada }\end{array}$ & $\begin{array}{l}\text { Motoshi Tomita } \\
\text { Japan }\end{array}$ \\
\hline $\begin{array}{l}\text { Kenta Shirasawa } \\
\text { Japan }\end{array}$ & $\begin{array}{l}\text { Brendan Stamper } \\
\text { USA }\end{array}$ & $\begin{array}{l}\text { Eleni Tsiplakou } \\
\text { Greece }\end{array}$ \\
\hline
\end{tabular}


Matthew Tucker

Australia

Christopher Tuggle

USA

Jennifer Tullet

UK

Jasim Uddin

Australia

Yoshinobu Uemoto

Japan

Giampiero Valè

Italy

Armand Valsesia

USA

Pim Van Hooft

Netherlands

Paul Vanraden

USA

Roel Veerkamp

Netherlands

Fabio Veronesi

Italy

Frédérique Viard

France

Marcelo Vicari

Brazil

Carlos Vicient

Spain

Joseph Vinetz

USA

Christof Vulsteke

Belgium

Patrik Waldmann

Sweden

Stefan Walter

USA

Kathryn Walters-Conte

USA

Ming-Bo Wang

Australia

Chaolong Wang

Singapore

Yaqun Wang

USA
Tao Wang

USA

Ning Wang

China

Tao Wang

USA

David Warrilow

Australia

Jonas Warringer

Sweden

Markus Weber

Switzerland

Karen Weck

USA

Zhi Wei

USA

Changshuai Wei

USA

Jochen Weishaupt

Germany

Emanuel Weitschek

Italy

Jared Westbrook

USA

Stephen White

USA

Kenneth Witwer

USA

Weiren Wu

China

Xifeng Wu

USA

Zheng Xia

USA

Yan Xiang

China

Zengyan Xie

China

Chao Xing

USA

Lizhen Xu

Canada

Yan Xu

China
Yong Xu

China

Haiming $\mathrm{Xu}$

China

Jun Xu

USA

Shawn Xu

USA

Lingyang Xu

USA

Peng Xu

China

Cheng Xue

USA

José Yáñez

Chile

Bing Yang

USA

Daichang Yang

China

Shengping Yang

USA

Chao-Yie Yang

USA

Yao Yang

USA

Guoyou Ye

Australia

Bin Yi

China

Jiye Yin

China

Hunter Young

USA

Neil Youngson

Australia

Bing Yu

USA

Paolo Zambonelli Italy

Mehdi Zarrei

Canada

Weiwei Zhai

Singapore 
Wenguang Zhang

China

Liangsheng Zhang

China

Fengyu Zhang

China

Deqiang Zhang

China

Yuan-Ming Zhang

China

Peng Zhang

USA

Wei Zhang

USA
Xinyu Zhang

USA

Xiquan Zhang

China

Tongwu Zhang

USA

Xueyong Zhang

China

Yong Zhang

China

M Zhang

China

Chen Zhao

China
Ni Zhao

USA

Jinshun Zhao

China

Min Zhong

USA

Xiang Zhou

USA

Ling Zhou

China

Jun Zhu

China

Jieyun Zhuang

China 